\title{
On Shapiro's Cyclic Inequality for $N=13$
}

\author{
By B. A. Troesch
}

\begin{abstract}
A cyclic sum $S(\mathbf{x})=\sum x_{i} /\left(x_{i+1}+x_{i+2}\right)$ is formed with the $N$ components of a vector $\mathbf{x}$, where $x_{N+1}=x_{1}, x_{N+2}=x_{2}$, and where all denominators are positive and all numerators are nonnegative. It is known that there exist vectors $\mathbf{x}$ for which $S(\mathbf{x})<N / 2$ if $N \geqslant 14$ and even, and if $N \geqslant 25$. It has been proved that the inequality $S(x) \geqslant N / 2$ holds for $N \leqslant 12$. Although it has been conjectured repeatedly that the inequality also holds for odd $N$ between 13 and 23 . this has apparently not yet been proved. Here we will confirm that the inequality indeed holds for $N=13$.
\end{abstract}

1. Introduction. In his book Analytic Inequalities [9], Mitrinovic describes the interesting history of a problem suggested by H. S. Shapiro [13]. The conjecture that the solution should exhibit a symmetric structure seemed reasonable, considering the near-symmetry of the problem, but the true solution turned out to be more subtle.

A cyclic sum $S(\mathbf{x})=\sum x_{i} /\left(x_{i+1}+x_{i+2}\right)$ is formed with the $N$ components of a vector $\mathbf{x}$, where $x_{N+1}=x_{1}, x_{N+2}=x_{2}$, and where all denominators are positive and all numerators nonnegative. The vector $\mathbf{x}$ with all components $x_{k}=1$ furnishes $S=N / 2$. If, for all $N$ and all $\mathbf{x}$, the inequality $S(\mathbf{x}) \geqslant N / 2$ were to hold, the problem would probably not have attracted particular attention (see its mention in [7]). However, Lighthill [5] showed that the inequality does not hold for $N=20$, and Zulauf sharpened this result to $N=14$ [14]. On the other hand, Mordell [10] conjectured that a nonsymmetric $\mathbf{x}$ could be found for $N \geqslant 7$ such that $S(\mathbf{x})<N / 2$. In steps the inequality was proved for $N=8$ [4], $N=10$ [11], and $N=12$ [6], and then it follows from a general property [3] that $S \geqslant N / 2$ is true for $N \leqslant 12$. For odd $N$ the counterexamples turned out to be harder to come by, but they were found down to $N=25$ [1], [2], [8]. The reason for the difference between odd and even $N$ 's was explained in [12]. Although it has been conjectured repeatedly that the inequality also holds for odd $N$ between 13 and 23, this has apparently not yet been proved. Here we will confirm that the inequality indeed holds for $N=13$.

For odd $N$ between 13 and 23 there is apparently no general method available to prove the inequality except the approach used by Nowosad [11] and Godunova and Levin [6]: One has to consider separately all the cases where the zero components of the vector $\mathbf{x}$ appear in various positions. For large $N$ the number of possibilities increases very rapidly, as the analysis in [11] and [6] shows. However, the number of cases to be discussed can be reduced significantly by taking advantage of two

Received July 12, 1984.

1980 Mathematics Subject Classification. Primary 25D15; Secondary 10C25, 52A40.

Ker words and phrases. Cyclic inequality, cyclic sum, minimization.

(C)1985 American Mathematical Society $0025-5718 / 85 \$ 1.00+\$ .25$ per page 
additional facts stated in Sections 2 and 3. Whereas the basic result of Diananda [3] permits the conclusion that the inequality is satisfied for $N=11$ because of the result in [6], it does not decide the question for odd $N \geqslant 13$.

There exists at most one relative minimum for every case. This result, derived in [11], is important because the problem is nonlinear and is solved by minimizing the sum $S$ for each case. Of course, when the stationary point is shown to be unique, it need not be checked for a minimum, because the boundary of the domain, i.e., $x_{k} \rightarrow 0$, is treated in connection with another case, and the sum $S(\mathbf{x})$ must only be checked against $N / 2$. There is also the possibility that no stationary point exists. If this cannot be proved, or if the statiunary points found are not at a relative minimum, then no decision is reached. This means that it is not a priori clear that a decision can be reached in all cases, regardless of the effort spent, say, in a numerical search.

The different cases are best characterized by listing the number of nonzero components of $\mathbf{x}$ after each zero. At the same time, only the nonvanishing terms of $\mathbf{x}$ will be numbered. For example, the notation $(4,1,5)$ represents a case for $N=13$, namely,

$$
x_{1} 0 x_{2} x_{3} x_{4} x_{5} 0 x_{6} 0 x_{7} x_{8} x_{9} x_{10}
$$

with the sum

$$
\begin{aligned}
S= & \left\{\frac{x_{2}}{x_{3}+x_{4}}+\frac{x_{3}}{x_{4}+x_{5}}+\frac{x_{4}}{x_{5}}+\frac{x_{5}}{x_{6}}\right\}+\left\{\frac{x_{6}}{x_{7}}\right\} \\
& +\left\{\frac{x_{7}}{x_{8}+x_{9}}+\frac{x_{8}}{x_{9}+x_{10}}+\frac{x_{9}}{x_{10}+x_{1}}+\frac{x_{10}}{x_{1}}+\frac{x_{1}}{x_{2}}\right\} .
\end{aligned}
$$

The three segments are $x_{2} x_{3} x_{4} x_{5}, x_{6}, x_{7} x_{8} x_{9} x_{10} x_{1}$.

2. The Pivotal Ratios. Let us call the group of terms between two zeros a segment. For instance, the example above consists of a 4-segment, a 1-segment, and a 5-segment. A 7-segment would be

$$
\cdots x_{1} 0 x_{2} x_{3} x_{4} x_{5} x_{6} x_{7} x_{8} 0 x_{9} \cdots
$$

with the segment sum

$$
S_{7}=\frac{x_{2}}{x_{3}+x_{4}}+\frac{x_{3}}{x_{4}+x_{5}}+\frac{x_{4}}{x_{5}+x_{6}}+\frac{x_{5}}{x_{6}+x_{7}}+\frac{x_{6}}{x_{7}+x_{8}}+\frac{x_{7}}{x_{8}}+\frac{x_{8}}{x_{9}} .
$$

At a stationary value of $S$, the leading ratios of all segments have the same value. This is easy to see by considering as a typical example the 7-segment. Introducing in the usual manner [6], [11] the vector $y$ by

$$
y_{1}=x_{2}, \quad y_{2}=x_{3}+x_{4}, \quad y_{3}=x_{4}+x_{5}, \ldots, y_{8}=x_{9},
$$

we have

$$
\begin{aligned}
S_{7}= & \frac{y_{1}}{y_{2}}+\frac{y_{2}+y_{4}-y_{5}+y_{6}-y_{7}}{y_{3}}-1+\frac{y_{3}+y_{5}-y_{6}+y_{7}}{y_{4}}-1 \\
& +\frac{y_{4}+y_{6}-y_{7}}{y_{5}}-1+\frac{y_{5}+y_{7}}{y_{6}}-1+\frac{y_{6}}{y_{7}}-1+\frac{y_{7}}{y_{8}} .
\end{aligned}
$$


For a stationary value, the vanishing partial derivatives $\partial S / \partial y_{k}$ give

$$
\begin{aligned}
-y_{1} / y_{2}+y_{2} / y_{3} & =0 \\
-y_{2} / y_{3}-y_{4} / y_{3}+y_{5} / y_{3}-y_{6} / y_{3}+y_{7} / y_{3}+y_{3} / y_{4} & =0, \\
y_{4} / y_{3}-y_{3} / y_{4}-y_{5} / y_{4}+y_{6} / y_{4}-y_{7} / y_{4}+y_{4} / y_{5} & =0, \\
-y_{5} / y_{3}+y_{5} / y_{4}-y_{4} / y_{5}-y_{6} / y_{5}+y_{7} / y_{5}+y_{5} / y_{6} & =0 \\
y_{6} / y_{3}-y_{6} / y_{4}+y_{6} / y_{5}-y_{5} / y_{6}-y_{7} / y_{6}+y_{6} / y_{7} & =0 \\
-y_{7} / y_{3}+y_{7} / y_{4}-y_{7} / y_{5}+y_{7} / y_{6}-y_{6} / y_{7}+y_{7} / y_{8} & =0 \\
-y_{7} / y_{8}+y_{8} / y_{9} & =0 .
\end{aligned}
$$

The last equation follows from the observation that the next segment starts with the simple ratio $y_{8} / y_{9}$.

By adding up all the middle equations we find that, denoting by $u$ the common ratio of the first and of the last terms in each segment,

$$
u=y_{1} / y_{2}=y_{2} / y_{3}=y_{7} / y_{8}=y_{8} / y_{9} \text {. }
$$

This result is very helpful in the following way. In the example above, the case $(4,1,5)$ is spelled out; another case would be $(1,4,5)$. But the solution of the nonlinear system must be the same, because if the leading terms are identical the solution for the stationary vector $\mathbf{y}$ is also the same, except for the interchanged indices. Therefore, among all the possible combinations only those with increasing segment length need to be considered. For $N=10$ and $N=12$ this is of little significance, but the larger the $N$, the more important this observation becomes.

3. A Reduction in the Number of Unknowns. Nowosad [11] has proved the following helpful result. If the relation between $\mathbf{x}$ and $\mathbf{y}$, as introduced above, is written as $\mathbf{x}=A \mathbf{y}$, and the matrix $A$ is symmetric with respect to the second diagonal or can be put into such a form by a cyclic shift, then the cyclic sum is not altered by the transformation $\eta=B \mathbf{y}$, where $B$ is the zero matrix, except for ones in the second diagonal. In other words, $S(\eta)=S(\mathbf{y})$ everywhere-in particular, at the stationary points and in their neighborhoods. This fact, together with the theorem that $S(\mathbf{y})$ can have at most one relative minimum for $y_{k}>0$, furnishes important relations between the components of $\mathbf{y}$.

We want to show that a modification of this property can be derived in all cases, without the assumption of the second diagonal symmetry. The property will be explained with a specific example, but the general proof would follow the same line of argument. In Section 2 we have considered the example of a segment of length 7; the $y_{2}$ to $y_{7}$ appear only in this particular segment, and the coupling to the neighboring segments is accomplished by $y_{1}$ and $y_{8}$.

We now define $\eta$ by the nonlinear transformation $\boldsymbol{\eta}=T \mathbf{y}$ :

$$
\begin{aligned}
\eta_{1} & =y_{1}, \\
\eta_{2} & =y_{2} y_{8} / y_{8}=y_{2}, \\
\eta_{3} & =y_{2} y_{8} / y_{7}, \\
& \cdots \\
\eta_{7} & =y_{2} y_{8} / y_{3}, \\
\eta_{8} & =y_{2} y_{8} / y_{2}=y_{8} .
\end{aligned}
$$


Then, with $y_{j}$ the leading element of the following segment,

$$
\begin{aligned}
\eta_{9} & =y_{9} y_{j} / y_{j}=y_{9}, \\
\eta_{10} & =y_{9} y_{j} / y_{j-1},
\end{aligned}
$$

Since the coupling elements $y_{1}$ and $y_{9}$ remain unchanged, the transformation $T$ can be carried out on each segment separately. Inserting the $y$, we obtain

$$
\begin{aligned}
S_{7}(\eta)= & \eta_{1} / \eta_{2}+\eta_{7}\left(1 / \eta_{8}-1 / \eta_{7}+1 / \eta_{6}-1 / \eta_{5}+1 / \eta_{4}-1 / \eta_{3}\right) \\
& +\eta_{6}\left(1 / \eta_{7}-1 / \eta_{6}+1 / \eta_{5}-1 / \eta_{4}+1 / \eta_{3}\right) \\
& +\eta_{5}\left(1 / \eta_{6}-1 / \eta_{5}+1 / \eta_{4}-1 / \eta_{3}\right) \\
& +\eta_{4}\left(1 / \eta_{5}-1 / \eta_{4}+1 / \eta_{3}\right)+\eta_{3}\left(1 / \eta_{4}-1 / \eta_{3}\right)+\eta_{2} / \eta_{3} .
\end{aligned}
$$

But this turns out to be exactly the same function as (2.1) for this and all other segments, and, hence,

$$
S(\mathbf{y})=S(T \mathbf{y}) .
$$

Since there is at most one relative minimum, we conclude that at such a point

$$
y_{2} y_{8}=y_{3} y_{7}=y_{4} y_{6}=y_{5} y_{5} \text {, }
$$

or, denoting the ratio $y_{k} / y_{k+1}$ by $r_{k}$,

$$
u=r_{2}=r_{7}, \quad r_{3}=r_{6}, \quad r_{4}=r_{5} \text {. }
$$

Quite generally, it can be shown for a $j$-segment that $u=r_{2}=r_{j}, r_{3}=r_{j-1}, \ldots$, and, finally,

$$
r_{j / 2}=r_{j / 2+2} \text { for } j \text { even, } \quad r_{j / 2+1 / 2}=r_{j / 2+3 / 2} \text { for } j \text { odd. }
$$

For segments up to length 5 (see below), these relations follow also directly from the equations for the stationary value. The number of unknowns is therefore reduced roughly by a factor of 2 .

4. Cases with Short Segments Only. We will show that $S<N / 2$ is possible only if one segment has at least length 6 . This is an extension of the known fact, based on the arithmetic-geometric mean inequality, that $S \geqslant N / 2$ if no segment is longer than 3.

A 1-segment has only one ratio $u$, and $S_{1}=u$; a 2-segment has $S_{2}=2 u$.

In the case of a 3-segment $\cdots x_{1} 0 x_{2} x_{3} x_{4} 0 x_{5} \cdots$, the sum is

$$
S_{3}=\frac{x_{2}}{x_{3}+x_{4}}+\frac{x_{3}}{x_{4}}+\frac{x_{4}}{x_{5}}=\frac{y_{1}}{y_{2}}+\frac{y_{2}-y_{3}}{y_{3}}+\frac{y_{3}}{y_{4}},
$$

and, at the stationary point,

$$
y_{1} / y_{2}=y_{2} / y_{3}=y_{3} / y_{4}=u \text {. }
$$

Since $x_{3}>0$, we obtain the result that $u>1$, which will be useful below.

The contribution of a 4-segment to the cyclic sum is

$$
\begin{aligned}
S_{4} & =x_{2} /\left(x_{3}+x_{4}\right)+x_{3} /\left(x_{4}+x_{5}\right)+x_{4} / x_{5}+x_{5} / x_{6} \\
& =y_{1} / y_{2}+\left(y_{2}+y_{4}\right) / y_{3}-1+y_{3} / y_{4}-1+y_{4} / y_{5} .
\end{aligned}
$$

For the stationary value we obtain

$$
\begin{gathered}
y_{1} / y_{2}=y_{2} / y_{3}, \quad-y_{2} / y_{3}-y_{4} / y_{3}+y_{3} / y_{4}=0, \\
y_{4} / y_{3}-y_{3} / y_{4}+y_{4} / y_{5}=0,
\end{gathered}
$$


and, hence, with the notation introduced above,

$$
-u+r_{3}-1 / r_{3}=0,
$$

or

$$
r_{3}=\left(u+\sqrt{u^{2}+4}\right) / 2>1 \text {. }
$$

The sum $S_{4}$ then becomes

$$
S_{4}=3 u-2+r_{3}+1 / r_{3}=3 u+\sqrt{u^{2}+4}-2 .
$$

After these preparations we now consider the cases where no $j$ is larger than 4 , with $k_{j}$ the number of $j$-segments, i.e., $\left\{k_{1}, k_{2}, k_{3}, k_{4}\right\}$. This corresponds to a total length of

$$
N=2 k_{1}+3 k_{2}+4 k_{3}+5 k_{4} \text {, }
$$

and the sum is

$$
S=k u+k_{4}\left(\sqrt{u^{2}+4}-2\right)-k_{3},
$$

where $k=k_{1}+2 k_{2}+3 k_{3}+3 k_{4}$.

The product of all ratios $r_{k}$ in the sum must equal unity, i.e.,

$$
u^{k} r_{3}^{k_{4}}=1
$$

It follows from (4.1) that

$$
u^{2 a}+u^{a+1}=1
$$

with $a=k / k_{4} \geqslant 3$. Clearly, then, $u<1$ and, furthermore,

$$
S>k u-k_{3} \text {. }
$$

In order to compare $S$ with $N / 2$ we evaluate

$$
\frac{S-N / 2}{k}>u-\frac{k_{3}}{k}-1+\frac{k_{2}}{2 k}+\frac{k_{3}}{k}+\frac{k_{4}}{2 k} \geqslant u-1+\frac{1}{2 a} \text {. }
$$

It can be shown in the following way that $u>1-1 / 2 a$. Since

$$
(1-x / \alpha)^{\alpha} \leqslant \exp (-x) \text { and } e^{-1}+e^{-1 / 2}<1,
$$

we conclude that

$$
(1-1 / 2 a)^{2 a}+(1-1 / 2 a)^{a+1}<(1-1 / 2 a)^{2 a}+(1-1 / 2 a)^{a}<1 .
$$

The comparison with (4.3) shows that $u>1-1 / 2 a$ and, therefore, $S>N / 2$.

The cases which include 5-segments as longest segments can also never lead to $S<N / 2$. At the stationary value we have

$$
\begin{gathered}
u=y_{1} / y_{2}=y_{2} / y_{3}=y_{5} / y_{6}, \quad-y_{2} / y_{3}-y_{4} / y_{3}+y_{5} / y_{3}+y_{3} / y_{4}=0, \\
y_{4} / y_{3}-y_{3} / y_{4}-y_{5} / y_{4}+y_{4} / y_{5}=0 .
\end{gathered}
$$

From the last equation, rewritten as

$$
r_{4}-r_{3}-1 / r_{4}+1 / r_{3}=\left(r_{4}-r_{3}\right)\left(1+1 / r_{3} r_{4}\right)=0,
$$

we obtain $r_{3}=r_{4}$, and, hence,

$$
-u+r_{3}-1 / r_{3}+1 / r_{3}^{2}=0 \text { or } u-1=\left(1-1 / r_{3}\right)^{2}\left(1+r_{3}\right) .
$$

If any 5-segment is present, we always have $u \geqslant 1$. 
Furthermore, all $x_{k}$ must be nonnegative; hence,

$$
x_{5}=y_{4}-y_{5}, \quad r_{3}=r_{4} \geqslant 1 .
$$

In the case described by $\left\{k_{1}, k_{2}, k_{3}, k_{4}, k_{5}\right\}$ the product of all $r$ 's is

$$
u^{k} r_{3,4}^{k} r_{3, \xi}^{k}=1 \text {, }
$$

where $k=k_{1}+2 k_{2}+3\left(k_{3}+k_{4}+k_{5}\right)$. (Here, the second subscript of $r_{i, j}$ refers to the $j$-segment.) If $k_{5} \neq 0$, then $r_{3,5} \geqslant 1$, and for the 4-segment $r_{3,4}>1$. This leaves only the possibility $u=1, r_{3.5}=1$-hence, a contradiction if $k_{4} \neq 0$, and if $k_{4}=0$, then $S=N / 2$.

The conclusion is therefore that any case with $S<N / 2$ must contain a segment of at least length 6. Even with a 6-segment present, some combinations can be readily eliminated, as we will show below.

5. General Properties of Segments of Lengths 6 and 7. For segments of length 6 , the unique relative minimum value, if it exists, can be found by a one-dimensional search. In order for the sum $S_{6}$ to be a minimum, we must have

$$
\begin{gathered}
u=r_{1}=r_{2}=r_{6}, \quad-u-1 / r_{3}+1 / r_{3} r_{4}-1 / r_{3}^{2} r_{4}+r_{3}=0, \\
1 / r_{3}-r_{3}-1 / r_{4}+1 / r_{3} r_{4}+r_{4}=0,
\end{gathered}
$$

where $r_{3}=r_{5}$ has been used and the redundant equation omitted. The last equation shows that $r_{4}<r_{3}$; from $x_{6}=y_{5}-y_{6} \geqslant 0$ and $x_{5}=y_{4}-y_{5}+y_{6} \geqslant 0$, it follows that $r_{3} \geqslant 1$ and $r_{4}-1+1 / r_{3} \geqslant 0$.

Linear combinations of the two equations above lead to

$$
\begin{gathered}
-u+2 / r_{3} r_{4}-1 / r_{3}^{2} r_{4}-1 / r_{4}+r_{4}=0, \\
-u r_{3}-1+1 / r_{3}-r_{3}+r_{3}^{2}+r_{4}=0,
\end{gathered}
$$

and we conclude that

$$
u r_{3}>r_{4}>u \text {. }
$$

In the case $\left\{k_{1}, k_{2}, k_{3}, k_{4}, k_{5}, k_{6}\right\}$ the product of all the ratios is

$$
p=u^{k} r_{3,4}^{k_{4}} r_{3,5}^{2 k_{5}} r_{3,6}^{2 k_{6}} r_{4,6}^{k_{6}}=1
$$

with $k=k_{1}+2 k_{2}+3\left(k_{3}+k_{4}+k_{5}+k_{6}\right)$.

Since the product of $r_{3,4}, r_{3,5}, r_{3,6}$ is larger than unity and $r_{4,6}>u$, it follows that $p>1$, if $k_{3}+k_{5}>0$. Therefore, no 3-segment or 5-segment can be present if the longest segments are of length 6 . In other words, the possibilities are restricted to $\left\{k_{1}, k_{2}, 0, k_{4}, 0, k_{6}\right\}$.

The resolution of a 6-segment leads to a one-dimensional search. One possibility is to carry it out by setting $r_{3}=\rho+\varepsilon, r_{4}=\rho-\varepsilon$, which leads to

$$
\rho^{2}=\varepsilon^{2}+1 / 2 \varepsilon-1, \quad 0 \leqslant \varepsilon<1 / 2 .
$$

From a given $\varepsilon$ we compute $\rho, r_{3}, r_{4}$, then $u$, and finally $S_{6}$. The sum $S_{6}$ is

$$
S_{6}=2\left(u+r_{3}+1 / r_{3}+r_{4}-2\right) \text {. }
$$

Therefore, from a simple table, for every $u$ (with increasing $\varepsilon, u$ decreases from $+\infty$ to 0 ) the 6-segment sum $S_{6}$ is determined, and also the product $p_{6}=u^{3} r_{3,6}^{2} r_{4,6}$ (see Table 1). 
TABLE 1

Short table for the case $(1,1,1,6)$

\begin{tabular}{lccccc}
\multicolumn{1}{c}{$\varepsilon$} & $r_{3}$ & $r_{4}$ & $u$ & $p$ & $S$ \\
0.05 & 3.0504 & 2.9504 & 2.7973 & & \\
0.10 & 2.1025 & 1.9025 & 1.7580 & & \\
0.15 & 1.6849 & 1.3849 & 1.2656 & & \\
0.20 & 1.4410 & 1.0410 & 0.9510 & & \\
0.2106 & 1.4016 & 0.9804 & 0.8967 & 1.00109 & 6.674355 \\
0.210625 & 1.4015 & 0.9803 & 0.8965 & 1.00000 & 6.673376 \\
0.2107 & 1.4013 & 0.9799 & 0.8962 & 0.99669 & 6.670410 \\
0.30 & 1.1699 & 0.5699 & 0.5329 & & \\
0.40 & 1.0403 & 0.2403 & 0.2341 & & \\
0.50 & 1.0000 & 0.0000 & 0.0000 & &
\end{tabular}

Similarly, the possibilities for segments of length 7 are also restricted. The sum $S_{7}$ is stationary if

$$
\begin{gathered}
-u-1 / r_{3}+1 / r_{3} r_{4}-1 / r_{3} r_{4}^{2}+1 / r_{3}^{2} r_{4}^{2}+r_{3}=0, \\
1 / r_{3}-r_{3}-1 / r_{4}+1 / r_{4}^{2}-1 / r_{3} r_{4}^{3}+r_{4}=0
\end{gathered}
$$

where $u=r_{7}, r_{3}=r_{6}, r_{4}=r_{5}$ have been used. A linear combination leads again to Eq. (5.2) and, hence, to

$$
u r_{3}>r_{4}
$$

The general case $\left\{k_{1}, k_{2}, \ldots, k_{7}\right\}$ has, as product of all terms,

$$
\prod_{j=1}^{j=7} p_{j}^{k^{\prime}} .
$$

If a 3- or 5-segment is present, then $u>1$, and, hence,

$$
\begin{aligned}
& p_{1}=u>1, \quad p_{2}=u^{2}>1, \quad p_{3}=u^{3}>1, \quad p_{4}=u^{3} r_{3,4}>1, \\
& p_{5}=u^{3} r_{3,5}^{2}>1, \quad p_{6}=u^{3} r_{3,6}^{2} r_{4,6}>1, \quad p_{7}=u^{3} r_{3,7}^{2} r_{4,7}^{2}>1 .
\end{aligned}
$$

We therefore conclude that no 3- or 5-segment can be present if the longest segment is of length 7 .

6. Segments of Length 6 or 7 if $N=13$. For $N=13$, the above results leave the case $(1,1,1,6)$, with $p=u^{3} p_{6}$ and $S=3 u+S_{6}$, as the only possibility with a 6-segment.

The numerical evaluation in Table 1 shows that $p$ decreases monotonically with $\varepsilon$, and for $p=1$ we obtain

$$
S=6.67337>13 / 2
$$

The case $(2,2,6)$ can easily be shown to lead to a cyclic sum $S$ which is never smaller than the cyclic sum for $(1,1,8)$, and this case is considered below.

As segments of length 7 , only the case $(4,7)$ need be considered, with $p=$ $u^{6} r_{3,4} r_{3,7}^{2} r_{4,7}^{2}=1$. However, we now show that

$$
p=r_{3,4}\left(u r_{3,7}\right)^{2}\left(u^{2} r_{4,7}\right)^{2}>r_{4,7}^{2}\left(u^{2} r_{4,7}\right)^{2}=\left(u r_{4,7}\right)^{4}>1 \text {. }
$$


From (4.2) we have $r_{3,4}>1$, and from (5.5), $u r_{3,7}>r_{4.7}$. Furthermore, (5.3) can be written as

$$
u r_{4}-1=\left(r_{3}-1\right)\left(r_{3} r_{4}-1 / r_{3} r_{4}+r_{4}-1\right) / r_{3}
$$

and, since $r_{3}>r_{4}>1, u r_{4,7}-1>0$. We conclude that, for $N=13$, the inequality $S \geqslant N / 2$ can possibly be violated only if one segment has at least length 8 .

7. The Remaining Cases for $N=13$. There are now only the cases $(1,1,8),(3,8)$, $(1,10)$, and (12) to be discussed. As Nowosad [11, p. 447] has shown, the case with all $x_{k} \neq 0$ need not be considered.

The case $(3,8)$ can be taken care of by a general result valid for any 8 -segment. There are three equations for the stationary value of $S$ :

$$
\begin{gathered}
-u-\frac{1}{r_{3}}+\frac{1}{r_{3} r_{4}}-\frac{1}{r_{3} r_{4} r_{5}}+\frac{1}{r_{3} r_{4}^{2} r_{5}}-\frac{1}{r_{3}^{2} r_{4}^{2} r_{5}}+r_{3}=0, \\
\frac{1}{r_{3}}-r_{3}-\frac{1}{r_{4}}+\frac{1}{r_{4} r_{5}}-\frac{1}{r_{4}^{2} r_{5}}+\frac{1}{r_{3} r_{4}^{2} r_{5}}+r_{4}=0, \\
-\frac{1}{r_{3} r_{4}}+\frac{1}{r_{4}}-r_{4}-\frac{1}{r_{5}}+\frac{1}{r_{4} r_{5}}-\frac{1}{r_{3} r_{4} r_{5}}+r_{5}=0,
\end{gathered}
$$

and the inequalities from the condition $x_{k}>0$ are

$$
r_{3}-1>0, \quad r_{4}-1+\frac{1}{r_{3}}>0, \quad r_{5}-1+\frac{1}{r_{4}}-\frac{1}{r_{3} r_{4}}>0 .
$$

Equation (7.2), written in the form

$$
r_{5}\left(r_{4}-r_{3}\right)\left(1+\frac{1}{r_{3} r_{4}}\right)=\frac{1}{r_{3} r_{4}^{2}}\left(-r_{3} r_{4}+r_{3}-1\right)<0
$$

furnishes the inequality $r_{4}<r_{3}$. Incidentally, it can be shown that this inequality is true for segments of any length.

Furthermore, the sum of Eqs. (7.1) and (7.2) leads to

$$
r_{4}-u=\frac{r_{3}-1}{r_{3} r_{4} r_{5}}\left\{r_{5}-1+\frac{1}{r_{4}}-\frac{1}{r_{3} r_{4}}\right\}>0
$$

or $r_{4}>u$.

A final useful relation, $r_{5}>u$, follows from the sum of all three equations above:

$$
-u-\frac{2}{r_{3} r_{4} r_{5}}+\frac{2}{r_{3} r_{4}^{2} r_{5}}-\frac{1}{r_{3}^{2} r_{4}^{2} r_{5}}+\frac{2}{r_{4} r_{5}}-\frac{1}{r_{4}^{2} r_{5}}-\frac{1}{r_{5}}+r_{5}=0,
$$

which is the same as

$$
\left(r_{5}-u\right) r_{3}^{2} r_{4}^{2} r_{5}=\left(r_{3} r_{4}-r_{3}+1\right)^{2}>0 .
$$

Considering the case $(3,8)$, we must have

$$
p=p_{3} p_{8}=u^{3} u^{3} r_{3}^{2} r_{4}^{2} r_{5}=1,
$$

and in a 3 -segment $u>1$, which leads to a contradiction if the inequalities above are taken into account. 


\begin{tabular}{cccc}
\multicolumn{5}{c}{ TABLE 2 } \\
& Case $(1,1,8)$ & Case $(1,10)$ & Case $(12)$ \\
$u$ & 0.865229 & 0.840551 & 0.822033 \\
$r_{3}$ & 1.300825 & 1.227239 & 1.164199 \\
$r_{4}$ & 0.965448 & 0.937842 & 0.906890 \\
$r_{5}$ & 1.307523 & 1.247627 & 1.723051 \\
$\lambda_{2}$ & 0.354429 & 0.203704 & 0.101057 \\
$S$ & 6.625060 & 6.601225 & 6.591599
\end{tabular}

The remaining cases $(1,1,8),(1,10)$, and $(12)$ have admissible stationary points, say $\mathbf{y}_{0}$. These points are readily found numerically. At the stationary points, the Hessian and its eigenvalues are computed. It turns out that in all three cases we have indeed found the (unique) relative minimum. The numerical values are listed in Table 2.

There always exists an eigenvalue $\lambda_{1}=0$ with eigenvector $\mathbf{y}_{0}$, because the function $S(\mathbf{y})$ is homogeneous of zero degree in $y_{k}$. All other eigenvalues at a relative minimum are positive, and $\lambda_{2}$, the smallest of them, is listed.

This completes the consideration of all possible cases and proves that, for $N=13$, $S(\mathbf{x}) \geqslant N / 2$ holds.

Department of Aerospace Engineering

University of Southern California

University Park

Los Angeles, California 90089-1454

1. P. J. Bushell \& A.H. Craven, "On Shapiro's cyclic inequality," Proc. Roy. Soc. Edinburgh, Sect. A, v. 26, 1975/76, pp. 333-338.

2. D. E. Da YKIN, "Inequalities for functions of a cyclic nature," J. London Math. Soc. (2), v. 3, 1971 , pp. 453-462.

3. P. H. Diananda, "On a cyclic sum," Proc. Glasgow Math. Assoc., v. 6, 1963, pp. 11-13.

4. D. Ž. DJokovic, "Sur une inégalité," Proc. Glasgow Math. Assoc., v. 6, 1963, pp. 1-10.

5. C. V. Durell, "Query,” Math. Gaz., v. 40, 1956, p. 266.

6. E. K. Godunova \& V. I. Levin, “A cyclic sum with 12 terms," Math. Notes, v. 19, 1976, pp. 510-517.

7. J. C. Lagarias, “The van der Waerden conjecture: Two Soviet solutions," Notices Amer. Math. Soc., v. 29,1982 , pp. 130-133.

8. M. A. Malcolm, “A note on a conjecture of L. J. Mordell,” Math. Comp., v. 25, 1971, pp. 375-377.

9. D. S. Mitrinovic, Analytic Inequalities, Springer-Verlag, New York, 1970, pp. 132ff.

10. L. J. MORDELL, "On the inequality $\sum_{r=1}^{n} x_{r} /\left(x_{r+1}+x_{r+2}\right) \geqslant \frac{1}{2} n$ and some others," Abh. Math. Sem. Univ. Hamburg, v. 22, 1958, pp. 229-240.

11. P. NowoSAD, "Isoperimetric eigenvalue problems in algebra," Comm. Pure Appl. Math., v. 21, 1968, pp. 401-465.

12. J. L. Searcy \& B. A. Troesch, "A cyclic inequality and a related eigenvalue problem," Pacific $J$. Math., v. 81, 1979, pp. 217-226.

13. H. S. Shapiro, "Problem 4603," Amer. Math. Monthly, v. 61, 1954, p. 571.

14. A. Zulauf, "Note on a conjecture of L. J. Mordell," Abh. Math. Sem. Univ. Hamburg, v. 22, 1958, pp. $240-241$. 